\title{
Mutational load may predict risk of progression in patients with Barrett's oesophagus and indefinite for dysplasia: a pilot study
}

\author{
Arvind J Trindade, ${ }^{01}$ Matthew J McKinley, ${ }^{2}$ Mohammad Alshelleh, ${ }^{1}$ Gabriel Levi, ${ }^{3}$ \\ Molly Stewart, ${ }^{1}$ Kathy J Quinn, ${ }^{4}$ Rebecca M Thomas ${ }^{4}$
}

To cite: Trindade AJ, McKinley MJ, Alshelleh M, et al. Mutational load may predict risk of progression in patients with Barrett's oesophagus and indefinite for dysplasia: a pilot study. BMJ Open Gastro 2019;6:e000268. doi:10.1136/ bmjgast-2018-000268

Received 14 December 2018 Revised 3 January 2019 Accepted 12 January 2019
Check for updates

(C) Author(s) (or their employer(s)) 2019. Re-use permitted under CC BY-NC. No commercial re-use. See rights and permissions. Published by BMJ.

${ }^{1}$ Division of Gastroenterology, Zucker School of Medicine at Hofstra/Northwell, Long Island Jewish Medical Center, Northwell Health System, New Hyde Park, New York, USA ${ }^{2}$ Division of Gastroenterology, ProHEALTH Care Associates, Lake Success, New York, USA ${ }^{3}$ Division of Pathology, ProHEALTH Care Associates, Lake Success, New York, USA ${ }^{4}$ Division of Pathology, Zucker School of Medicine at Hofstra/ Northwell, Long Island Jewish Medical Center, Northwell Health System, New Hyde Park, New York, USA

Correspondence to Dr Arvind J Trindade; arvind.trindade@gmail.com

\section{ABSTRACT}

Background and aims Mutational load (ML) has been shown to help risk-stratify those that may progress from non-dysplastic Barrett's oesophagus (BE) to dysplastic disease. Management of patients with $\mathrm{BE}$ and indefinite for dysplasia (BE-IND) is challenging and risk stratification tools are lacking. The aim of this pilot study is to evaluate the utility of ML for risk stratification in patients with $\mathrm{BE}$ IND.

Methods This is a single-centre, retrospective pilot study evaluating $\mathrm{ML}$ quantification in patients with $\mathrm{BE}-$ IND. Histology at follow-up endoscopy at least 1 year after the baseline endoscopy was used to determine if a patient progressed to low or high dysplasia. The ML levels were then compared among patients who progressed to dysplasia versus those who did not.

Results Thirty-five patients who met the inclusion criteria were identified, and seven met the exclusion criteria. Twenty-eight patients were analysed, of whom eight progressed to low-grade dysplasia (6) and high-grade dysplasia (2). Seven of these eight patients had some level of genomic instability detected in their IND biopsy (ML $\geq 0.5)$. Ten of the 20 (50\%) who did not progress had no ML level. At an ML cut-off above 1.5, the risk of progression to high-grade dysplasia was $33 \%$ vs $0 \%$ $(p=0.005)$, with a sensitivity of $100 \%$ and a specificity of $85 \%$.

Conclusion These results indicate that ML may be able to risk-stratify progression to high-grade dysplasia in BE-IND. Larger studies are needed to confirm these findings.

\section{INTRODUCTION}

Barrett's oesophagus (BE) is defined as a change from normal oesophageal squamous epithelium to metaplastic columnar epithelium with goblet cells, usually in association with gastro-oesophageal reflux disease. ${ }^{1}$ It is a major risk factor for oesophageal adenocarcinoma. Despite recommendations for screening and surveillance in Barrett's, the incidence of oesophageal adenocarcinoma continues to rise. ${ }^{2}$ It is estimated that $5.6 \%$ of the adult population have $\mathrm{BE}$ in the USA. ${ }^{1}$ The

\section{Summary box}

What is already known about this subject?

- Mutational load has been shown to help risk-stratify those that may progress from non-dysplastic Barrett's oesophagus to dysplastic disease.

- Management of patients with Barrett's oesophagus and indefinite for dysplasia is challenging and risk stratification tools are lacking.

What are the new findings?

- This pilot study shows that mutational load may be able to risk-stratify which patients progress to highgrade dysplasia.

How might it impact on clinical practice in the foreseeable future?

If our results are reproducible in large studies, then mutational load may be an option to risk-stratify patients with Barrett's oesophagus and indefinite for dysplasia.

only currently used biomarker for risk stratification of $\mathrm{BE}$ is dysplasia. However finding and appropriately classifying dysplasia can be difficult. Often dysplasia is focal and finding it can be challenging given most sampling techniques sample a minority of the Barrett's mucosa. Dysplasia in BE has been classified in a three-tier system as follows: indefinite for dysplasia (IND), low-grade dysplasia (LGD), and high-grade dysplasia (HGD).$^{34}$ IND is a category where the observed architectural and nuclear abnormalities are less diagnostic than those seen in clear-cut dysplasia, or when there are significant architectural/cytological atypical features but also significant concomitant inflammation such that reactive atypia cannot be excluded. In clinical practice LGD and IND are often treated the same way in regard to surveillance. ${ }^{5}$ However, with the recent shift in the guidelines to ablation of LGD due to a randomised control 
trial showing benefit in regard to lack of progression to neoplasia, it is a bit unclear clinically how to manage IND patients. ${ }^{6}$ In addition, a recent multicentre study has shown that patients diagnosed with IND behave similarly to LGD in regard to biological behaviour. ${ }^{5}$ Triaging IND patients according to risk of future progression would help to limit unnecessary repeat endoscopies in patients at low risk and justify closer observation in patients at higher risk, perhaps even supporting early means of cancer prevention such as ablation.

Thus, other biomarkers may be helpful in risk stratification in BE-IND. Currently, dysplasia is the only biomarker used to risk-stratify BE. Despite its widespread use, it is unclear whether surveillance endoscopy to detect this biomarker is useful. ${ }^{8-11}$ As a result research for other biomarkers, particularly molecular biomarkers, to help risk-stratify $\mathrm{BE}$ is under way. ${ }^{12-15}$ One potential biomarker that has been identified is mutational load (ML) as a measure of genetic aberration and instability. ${ }^{16}$ ML provides a measure of cumulative genomic instability at 10 key genomic loci in patients with BE by assessing DNA damage in proximity to tumour suppressor genes associated with progression to HGD and EA. BE tissue with a higher degree of genetic aberrations, specifically loss of heterozygosity (LOH) of tumour suppressor genes, progresses to more advanced disease. ${ }^{17}$

ML assessment can be determined using a commercially available test (BarreGEN, Interpace Diagnostics, Pittsburgh, Pennsylvania, USA). The test quantifies the degree of cumulative genetic derangement of 10 genetic loci of tumour suppressor genes, specifically assessing the presence of LOH mutations and new alleles consistent with microsatellite instability (MSI). The following genetic loci are tested, with their tumour suppressor genes in parentheses: 1p (CMM1, L-myc), 3p (VHL, HoGG1), 5q (MCC, APC), 9p (CDKN2A), 10q (PTEN, MXI1), 17p (TP53), 17q (RNF43, NME1), 18q (SMAD4, DCC), 21q (TFF1, PSEN2) and 22q (NF2). ${ }^{16} 18$ All LOH mutations are assigned a numerical value based on the degree of derangement. ML consists of a statistically derived weighted scoring system from 0 to 10 , with 0 representing the lowest level of genomic instability and 10 representing the highest level of genomic instability. ${ }^{16} 19$

A recent case-control study evaluated the utility of ML in predicting progression to neoplasia (HGD or intramucosal cancer) based on samples of only BE with LGD or non-dysplastic BE at baseline. ${ }^{16}$ Cases that progressed to neoplasia on follow-up (23 patients) were compared with controls (46 patients who did not progress to neoplasia). The mean ML was higher in cases that progressed than controls (2.21 vs $0.42, \mathrm{p}<0.001)$. The study concluded that ML in preprogression tissue can predict progression to neoplasia in BE and thus may serve as a useful biomarker in surveillance of $\mathrm{BE}$.

ML is proportional to the degree of dysplasia, and thus may serve as an adjunctive test in patients with equivocal histology. A retrospective study looked at 271 patients with varying degrees of dysplasia (IND, LGD and HGD). ${ }^{18}$
The authors found that the ML correlated to the grade of dysplasia (1.1 vs 2.2 vs 3.3, respectively; correlation coefficient $=0.60, \mathrm{p}<0.0001)$. The authors concluded that $\mathrm{ML}$ may be a useful adjunct to histological evaluation. Another retrospective study examined 877 microdissected targets from BE biopsies. Increasing ML correlated to increasing severe histology in regard to grade of dysplasia (correlation coefficient $=0.68, \mathrm{p}<0.0001){ }^{19}$

The aim of this study is to determine if ML can help risk-stratify patients with BE-IND.

\section{METHODS}

This is a retrospective review of patients diagnosed with BE-IND at North Shore University Hospital from 2013 to 2017. A prospectively maintained database was searched for consecutive patients. The inclusion criteria included (1) diagnosis of BE-IND on an endoscopic exam without a concurrent diagnosis of true dysplasia elsewhere in the oesophagus; (2) underwent endoscopic surveillance for IND with Seattle protocol biopsies ${ }^{20}$; (3) underwent ML testing for risk stratification on the index endoscopy biopsies showing IND (preprogression tissue); and (4) had adequate follow-up of at least 1 year if no dysplasia was detected on subsequent exams. Exclusion criteria included the following: (1) underwent endoscopic ablation; (2) lack of 1-year follow-up for patients who did not have dysplasia on a follow-up exam; (3) history of LGD or HGD; (4) dysplasia developing within 1 year of the initial endoscopy (as the dysplasia was likely present on the index exam); (5) presence of oesophagitis on histology or endoscopy; and (6) patient not on a medical antacid regimen.

ML testing was performed on baseline IND biopsy tissue blinded to the future progression status of patients (BarreGEN, Interpace Diagnostics). All cases in this series were re-examined by the pathologist and only targets that contained the IND were used. ML was measured using the formalin-fixed, paraffin-embedded (FFPE) tissue from biopsies taken at the time of baseline endoscopy. H\&E-stained FFPE slides were microscopically examined by pathologists to identify representative areas of IND histology. H\&E-stained slides were used to guide microdissection of recut, unstained, $4 \mu \mathrm{m}$ thick, FFPE slides. Slides were microdissected for the maximum number of histological targets with IND available for each patient. Microdissection was performed manually, targeting areas in which epithelial cells constituted $90 \%$ or more of the total cells removed. By microscopic estimation, no more than $10 \%$ of microdissected cells were stromal or inflammatory cells. Accuracy of all microdissections was carefully reviewed by two pathologists.

Selected areas for microdissection contained mainly epithelial cells. DNA from the microdissected targets was then prepared. PCR and quantitative capillary electrophoresis of DNA were used to detect the presence of LOH and new alleles consistent with MSI of the selected DNA markers for the previously discussed 10 genetic loci 
of tumour suppressor genes. For each microdissected tissue, it was determined whether each $\mathrm{LOH}$ mutation is of low (50\%-75\% of the DNA contained $\mathrm{LOH}$ ) or high ( $>75 \%$ of the DNA contains LOH mutations) clonality. The sum of the clonality of each genetic loci is the ML. ${ }^{19}$

Two gastrointestinal pathologists (GL and RMT), with extensive experience in a high-volume Barrett's tertiary care referral centre, reviewed all initial IND histology and follow-up histology. For the purposes of this study, the histology was rereviewed to ensure IND was an accurate diagnosis prior to preparation for ML analysis. This rereview also served to help locate the area on the tissue where ML would be measured. It should be noted that in our institution, the current clinical practice is that any diagnosis of dysplasia including IND is reviewed at the gastrointestinal pathology consensus meeting, where three to five gastrointestinal pathologists are present.

Patient characteristics were abstracted from the medical chart. Data analysis was separated into two groups. The first group was subjects with BE and IND on the index endoscopy who developed dysplasia (LGD, HGD or intramucosal cancer) on subsequent follow-up at least 1 year after the index pathology of IND. The second group was subjects with BE and IND on the index endoscopy who did not develop dysplasia on subsequent follow-up at least 1 year after the index pathology of IND. These patients had non-dysplastic histology or continued IND histology on follow-up exams. The two groups were compared to determine if there is an ML cut-off that can predict progression to dysplasia or neoplasia (HGD/ intramucosal cancer) in the IND cohort within 1 year of the index endoscopy.

$\chi^{2}$ and Fisher's exact tests were used to compare categorical variables, and the Student's t-test was used for continuous variables. All analyses were conducted using SAS V.9.4.

\section{RESULTS}

Thirty-five patients who met the inclusion criteria were identified, and seven who met the exclusion criteria were excluded (three with oesophagitis, three without 1-year follow-up and one not on antacid medication). The study analysed 28 consecutive IND patients at baseline biopsy, $61 \%$ were male with a mean age of 64 years (table 1 ). All IND pathology was confirmed by the pathologists in this study without disagreement. All patients had 1-year follow-up endoscopy, with corresponding follow-up biopsy indicating no progression or indicating progression to LGD or HGD, with 22 patients $(79 \%)$ having 2 years and 15 patients (54\%) having 3 years of endoscopic surveillance follow-up. Of all IND patients, six eventually progressed to LGD (21\%) and two to HGD (7\%). The baseline mean BE segment length was similar in IND patients who later progressed to LGD $(4.0 \mathrm{~cm})$ versus those who did not $(3.7 \mathrm{~cm})$, but significantly longer in patients who progressed to HGD $(7.5 \mathrm{~cm})$. Of all patients with baseline IND, 29\% (8/28) progressed to LGD or HGD, while only 7\% (2/28) progressed to HGD.

For analysis, ML scores in IND biopsies were grouped into numerical categories shown in tables 2 and 3 . Many IND patients who did not progress to LGD or HGD lacked all detectable genomic instability $(10 / 20)$ (ML=0; table 2). By contrast $88 \%$ (7/8) of patients who progressed to LGD or HGD had at least some level of genomic instability detected in their IND biopsy (ML $\geq 0.5$ ). The sensitivity and specificity for identifying patients who would later progress to LGD or HGD at this ML threshold were $88 \%$ and $50 \%$, respectively (table 2). Using this ML threshold for genomic instability (ML $\geq 0.5$ ) separated patients who had an initial $29 \%$ risk of progression to LGD or HGD at baseline into two, more refined risk categories: (1) those at lower risk of progression to LGD or HGD (9\% risk of progression) and (2) those at higher risk of progression to LGD or HGD ( $41 \%$ risk of progression) (9\% vs $41 \%, \mathrm{p}=0.07$ ). Higher levels of genomic instability provided higher specificity for predicting which IND patients would progress but at the expense of lower sensitivity for progression.

In contrast, patients who progressed to HGD had comparably higher levels of genomic instability at baseline IND biopsy ( $\mathrm{ML} \geq 1.5$; table 3 ). The sensitivity and specificity for identifying patients who would later progress to HGD at this ML threshold were $100 \%$ and $85 \%$, respectively. Using this ML threshold for genomic

Table 1 Patient demographics

\begin{tabular}{lllll}
\hline & $\begin{array}{l}\text { IND progressed to } \\
\text { HGD } \\
\mathbf{n = 2}\end{array}$ & $\begin{array}{l}\text { IND progressed to } \\
\text { LGD } \\
\end{array}$ & $\begin{array}{l}\text { No IND } \\
\text { progression } \\
\mathbf{n = 2 0}\end{array}$ & $\begin{array}{l}\text { All IND } \\
\mathbf{n = 2 8}\end{array}$ \\
\hline Age (years), mean & 57 & 61.17 & 65.2 & 63.75 \\
\hline Sex (male) & $2 / 2(100 \%)$ & $5 / 6(83.33 \%)$ & $10 / 20(50 \%)$ & $17 / 28(60.71 \%)$ \\
Hiatal hernia & $1 / 2(50 \%)$ & $1 / 6(16.67 \%)$ & $7 / 20(35 \%)$ & $9 / 28(32.14 \%)$ \\
\hline Length mean (cm) & 7.5 & 4 & 3.68 & 4.04 \\
\hline Patients had 1-year follow-up exam & $2 / 2(100 \%)$ & $6 / 6(100 \%)$ & $20 / 20(100 \%)$ & $28 / 28(100 \%)$ \\
\hline 2-year follow-up & $2 / 2(100 \%)$ & $4 / 6(66.67 \%)$ & $16 / 20(80 \%)$ & $22 / 28(78.57 \%)$ \\
\hline 3-year follow-up & $2 / 2(100 \%)$ & $2 / 6(33.33 \%)$ & $11 / 20(55 \%)$ & $15 / 28(53.57 \%)$ \\
\hline
\end{tabular}

HGD, high-grade dysplasia; IND, indefinite for dysplasia; LGD, low-grade dysplasia. 


\begin{tabular}{llllll}
\hline Table 2 & ML performance in predicting future progression to LGD or HGD in IND patients at baseline \\
\hline & $\begin{array}{l}\text { No IND progression } \\
\text { LGD or HGD }\end{array}$ & $\begin{array}{l}\text { IND progressed to } \\
\text { LGD or HGD }\end{array}$ & $\begin{array}{l}\text { Specificity } \\
(\%)\end{array}$ & $\begin{array}{l}\text { Sensitivity } \\
\text { (\%) }\end{array}$ & $\begin{array}{l}\text { \% IND that progressed to LGD } \\
\text { or HGD }\end{array}$ \\
\hline$M L=0$ & 10 & 1 & NA & NA & $\begin{array}{l}\text { 9\% of IND progressed } \\
\text { (lower risk) }\end{array}$ \\
$M L=0.5-0.75$ & 3 & 3 & 50 & 88 & $41 \%$ of IND progressed \\
(higher risk)
\end{tabular}

HGD, high-grade dysplasia; IND, indefinite for dysplasia; LGD, low-grade dysplasia; ML, mutational load; NA, not available.

instability (ML $\geq 1.5$ ) separated patients who had an initial $7 \%$ risk of progression to HGD at baseline into two, more refined risk categories: (1) those at lower risk of progression to HGD ( $0 \%$ rate of progression) and (2) those at higher risk of progression to HGD (33\% rate of progression) ( $0 \%$ vs $33 \%, \mathrm{p}=0.005$ ). Again, higher levels of genomic instability provided higher specificity for predicting which IND patients would progress to HGD.

\section{DISCUSSION}

In this study we show that ML can help risk-stratify patients who may progress to dysplasia in patients with $\mathrm{BE}$ with IND. In this study $29 \%$ progressed to dysplasia (LGD and HGD) and 7\% progressed to HGD. This is in line with other single-centre series from tertiary care centre. Previous studies have shown an annual progression rate of $12.9 \%-25 \%$ depending on the series. ${ }^{21-23}$ Seven out of the eight patients who progressed to dysplasia in our study had some level of genomic instability. ML had been shown previously to help risk-stratify patients with non-dysplastic BE or with LGD in regard to progression to HGD. ${ }^{16}$ As discussed earlier, these IND patients can be difficult to manage and require more intense surveillance. Thus further risk stratification can be helpful in this subgroup of patients.

This study is novel in that it is the first to examine if ML can be a predictor of progression to true dysplasia in patients with BE and IND. Previous studies have shown ML to be a predictor for progression in non-dysplastic disease. ${ }^{1819}$ Other studies have looked at DNA content abnormalities from FFPE tissue, such as aneuploidy measured by flow cytometry, in predicting progression from IND to dysplasia. ${ }^{23} 24$ These studies show that DNA flow cytometry can risk-stratify patients with BE-IND who will progress to dysplasia.

Our results show that patients with an ML above 0.5 should be considered for more frequent surveillance compared with those with no ML, as these patients had a higher risk of progression to low-grade and high-grade dysplasia $(41 \%$ vs $9 \%, \mathrm{p}<0.07)$. The result was not significant likely due to the small sample size. On the other hand, an ML above 1.5 statistically predicted progression to HGD versus those with an ML below $1.5(33 \%$ vs $0 \%, \mathrm{p}=0.005$ ). Based on this, an ML above 1.5 should be strongly considered for frequent surveillance and perhaps even advanced imaging if no obvious lesions are visualised given the focal nature of dysplasia.

Not surprisingly, our results are more robust for risk stratification in regard to patients who eventually develop HGD compared with those patients who progressed to LGD. This could be related to the inherent issues with the classification of LGD, ${ }^{25-27}$ which include high interobserver variability among expert pathologists. A sensitivity and specificity of $100 \%$ and $85 \%$ for an ML above 1.5 for progression to HGD in this cohort support its use as a risk stratification tool in BE for progression to HGD.

The strengths of our study include a true IND cohort. Patients' histology from preprogression tissue was rereviewed by experts in gastrointestinal pathology. In addition patients were excluded if there were any signs of

Table $3 \mathrm{ML}$ performance in predicting future progression to HGD in IND patients at baseline

\begin{tabular}{|c|c|c|c|c|c|}
\hline & $\begin{array}{l}\text { No IND progression } \\
\text { to HGD }\end{array}$ & $\begin{array}{l}\text { IND progressed } \\
\text { to } H G D\end{array}$ & Specificity (\%) & Sensitivity (\%) & $\begin{array}{l}\text { \% IND that progressed to } \\
\text { HGD }\end{array}$ \\
\hline$M L=0$ & 11 & 0 & NA & NA & \multirow{3}{*}{$\begin{array}{l}0 \% \text { progressed } \\
\text { (low risk) }\end{array}$} \\
\hline$M L=0.5-0.75$ & 6 & 0 & 42 & 100 & \\
\hline$M L=1.0-1.25$ & 5 & 0 & 65 & 100 & \\
\hline$M L=1.5-1.75$ & 2 & 1 & 85 & 100 & \multirow[t]{2}{*}{$33 \%$ progressed (higher risk) } \\
\hline$M L \geq 2$ & 2 & 1 & 92 & 50 & \\
\hline Total & 26 & 2 & & & Overall $7 \%$ of IND progressed \\
\hline
\end{tabular}

HGD, high-grade dysplasia; IND, indefinite for dysplasia; ML, mutational load; NA, not available. 
oesophagitis on pathology or if patients were not optimised on a medical antacid regimen.

Our study does have limitations. This is a singlecentre, retrospective study. We decided to make this a single-centre study to control for heterogeneity in pathological classifications. It also afforded us the opportunity to allow our pathologists to review all baseline pathology. In addition the sample size is relatively small at 28 patients. Another limitation is that despite patients undergoing standard-of-care Seattle protocol biopsies, it is possible that LGD or HGD is missed on the initial exam if a dysplastic area was not seen on endoscopy and missed on random biopsies, especially since this cohort consists of patients with long-segment Barrett's. This is a known phenomenon that can occur in any surveillance programme. ${ }^{1128}$ Finally the follow-up period that was required for this study was only 1 year (54\% of patients had 3-year follow-up). It is possible that patients who had ML levels above 0.5 and did not progress may progress to low-grade or high-grade dysplasia on further follow-up.

Despite the limitations our pilot study shows that ML may be a useful test for risk stratification in BE-IND. It should be noted that we are not advocating ablation based on ML levels. The decision for ablation should be dictated by the presence of dysplasia, as per the guidelines. However we do feel that ML can dictate surveillance intervals and thus help risk-stratify patients. Larger studies in the future on a BE-IND cohort may give more insight into the sensitivity and specificity of the test. In our study we had one patient with a high ML $(>2)$ with over 3 years of follow-up who never developed dysplasia. On the other hand, we had a patient who developed LGD who never had a measurable ML. Given the small cohort, this affects the sensitivity and specificity of the test, per table 2. Larger studies would be able to account for these extremes and give a better estimate of the sensitivity and specificity of ML in predicting progression to dysplasia. Despite the small numbers it seems that the sensitivity and specificity for development of HGD at ML of 1.5-1.75 $(100,85 \%)$ hold promise to be a possible indicator of BE-IND to develop to HGD. Larger prospective observational studies are needed to confirm our findings given only two patients in the cohort progressed to HGD.

In summary, IND patients had a low risk of progression to HGD but a significantly higher risk of progression to any to type of dysplasia (LGD or HGD). Genomic instability can further refine risk in these patients by dividing them into categories: (1) those at lower risk of progression to dysplasia and (2) those at higher risk of progression to dysplasia compared with the risk initially conferred by their baseline pathology diagnosis of IND alone. Our results are consistent with those of previous studies demonstrating that ML can be a useful biomarker in identifying patients with $\mathrm{BE}$ at risk of future progression to EA, allowing for closer surveillance or cancer preventative treatment in patients at higher risk of progression and avoiding unnecessary interventions in those at lower risk.
Contributors Conception and design: AJT, RMT. Analysis and interpretation of the data: AJT, MJM, MA, GL, MS, KJQ, RMT. Drafting of the article: AJT, MJM, MA, GL, MS, KJQ, RMT. Critical revision of the article for important intellectual content: AJT, MJM, MA, GL, MS, KJQ, RMT. Final approval of the article: AJT, MJM, MA, GL, MS, KJQ, RMT.

Funding The authors have not declared a specific grant for this research from any funding agency in the public, commercial or not-for-profit sectors. Interpace Diagnostics kindly supported the open access charges for this article. Interpace was not involved in the trial design, conduct of the trial, or preparation of the manuscript.

Competing interests None declared.

Patient consent for publication Not required.

Ethics approval The study was approved by the Zucker School of Medicine Institutional Review Board. All research was performed in accordance with all relevant guidelines/regulations and conforms to the ethical guidelines of the 1975 Declaration of Helsinki. Informed consent was obtained from all participants.

Provenance and peer review Not commissioned; externally peer reviewed.

Data sharing statement Specific requests for data included in this study can be made to Arvind.trindade@gmail.com.

Open access This is an open access article distributed in accordance with the Creative Commons Attribution Non Commercial (CC BY-NC 4.0) license, which permits others to distribute, remix, adapt, build upon this work non-commercially, and license their derivative works on different terms, provided the original work is properly cited, appropriate credit is given, any changes made indicated, and the use is non-commercial. See: http://creativecommons.org/licenses/by-nc/4.0/.

\section{REFERENCES}

1. Spechler SJ, Souza RF. Barrett's esophagus. N Engl J Med Overseas Ed 2014;371:836-45.

2. Pohl $\mathrm{H}$, Welch $\mathrm{HG}$. The role of overdiagnosis and reclassification in the marked increase of esophageal adenocarcinoma incidence. $J$ Natl Cancer Inst 2005;97:142-6.

3. Odze RD. Update on the diagnosis and treatment of Barrett esophagus and related neoplastic precursor lesions. Arch Pathol Lab Med 2008;132:1577-85.

4. Reid BJ, Haggitt RC, Rubin CE, et al. Observer variation in the diagnosis of dysplasia in Barrett's esophagus. Hum Pathol 1988;19:166-78.

5. Sinh P, Anaparthy R, Young PE, et al. Clinical outcomes in patients with a diagnosis of "indefinite for dysplasia" in Barrett's esophagus: a multicenter cohort study. Endoscopy 2015;47:669-74.

6. Shaheen NJ, Falk GW, Iyer PG, et al. ACG clinical guideline: diagnosis and management of Barrett's esophagus. Am J Gastroenterol 2016;111:30-50.

7. Phoa KN, van Vilsteren FG, Weusten BL, et al. Radiofrequency ablation vs endoscopic surveillance for patients with Barrett esophagus and low-grade dysplasia: a randomized clinical trial. JAMA 2014;311:1209-17.

8. Choi SE, Hur C. Screening and surveillance for Barrett's esophagus: current issues and future directions. Curr Opin Gastroenterol 2012;28:377-81.

9. Spechler SJ. Barrett esophagus and risk of esophageal cancer: a clinical review. JAMA 2013;310:627-36.

10. Falk GW, Ours TM, Richter JE. Practice patterns for surveillance of Barrett's esophagus in the United States. Gastrointest Endosc 2000;52:197-203.

11. Corley DA, Mehtani K, Quesenberry C, et al. Impact of endoscopic surveillance on mortality from Barrett's esophagus-associated esophageal adenocarcinomas. Gastroenterology 2013;145:312-9.

12. Jankowski M, Wani S. Diagnostic and management implications of basic science advances in Barrett's Esophagus. Curr Treat Options Gastroenterol 2015;13:16-29.

13. Matsuzaki J, Suzuki H. Circulating microRNAs as potential biomarkers to detect transformation of Barrett's oesophagus to oesophageal adenocarcinoma. BMJ Open Gastroenterol 2017;4.

14. Matsuzaki J, Suzuki H. MicroRNAs in Barrett's esophagus: future prospects. Front Genet 2014;5.

15. Matsuzaki J, Suzuki $H$, Tsugawa $H$, et al. Bile acids increase levels of microRNAs 221 and 222, leading to degradation of CDX2 during esophageal carcinogenesis. Gastroenterology 2013;145:1300-11.

16. Eluri S, Brugge WR, Daglilar ES, et al. The presence of genetic mutations at key loci predicts progression to esophageal 
adenocarcinoma in Barrett's esophagus. Am J Gastroenterol 2015;110:828-34.

17. Lin X, Finkelstein SD, Zhu B, et al. Loss of heterozygosities in Barrett esophagus, dysplasia, and adenocarcinoma detected by esophageal brushing cytology and gastroesophageal biopsy. Cancer 2009;117:57-66

18. Ellsworth E, Jackson SA, Thakkar SJ, et al. Correlation of the presence and extent of loss of heterozygosity mutations with histological classifications of Barrett's esophagus. BMC Gastroenterol 2012;12.

19. Khara HS, Jackson SA, Nair S, et al. Assessment of mutational load in biopsy tissue provides additional information about genomic instability to histological classifications of Barrett's esophagus. J Gastrointest Cancer 2014;45:137-45.

20. Vaezi MF, Pandolfino JE, Vela MF. ACG clinical guideline: diagnosis and management of achalasia. Am J Gastroenterol 2013;108:1238-49.

21. Ma M, Shroff S, Feldman M, et al. Risk of malignant progression in Barrett's esophagus indefinite for dysplasia. Dis Esophagus 2017;30:1-5.

22. Horvath $\mathrm{B}$, Singh $\mathrm{P}$, Xie H, et al. Risk for esophageal neoplasia in Barrett's esophagus patients with mucosal changes indefinite for dysplasia. J Gastroenterol Hepatol 2015;30:262-7.
23. Choi W-T, Emond MJ, Rabinovitch PS, et al. "Indefinite for Dysplasia" in Barrett's esophagus: inflammation and dna content abnormality are significant predictors of early detection of neoplasia. Clin Trans Gastroenterol 2015;6:e81.

24. Choi W-T, Tsai J-H, Rabinovitch PS, et al. Diagnosis and risk stratification of Barrett's dysplasia by flow cytometric DNA analysis of paraffin-embedded tissue. Gut 2018;67:1229-38.

25. Duits LC, van der Wel MJ, Cotton CC, et al. Patients with Barrett's esophagus and confirmed persistent low-grade dysplasia are at increased risk for progression to Neoplasia. Gastroenterology 2017;152:993-1001.

26. Kerkhof M, van Dekken H, Steyerberg EW, et al. Grading of dysplasia in Barrett's oesophagus: substantial interobserver variation between general and gastrointestinal pathologists. Histopathology 2007;50:920-7.

27. Montgomery E, Bronner MP, Goldblum JR, et al. Reproducibility of the diagnosis of dysplasia in Barrett esophagus: a reaffirmation. Hum Pathol 2001;32:368-78.

28. Sharma P, Falk GW, Weston AP, et al. Dysplasia and cancer in a large multicenter cohort of patients with Barrett's esophagus. Clin Gastroenterol Hepatol 2006;4:566-72. 